\title{
Micromachining of Transparent Materials with Fresnel Diffraction of Infrared Radiation
}

\author{
K.Okazaki $^{* 1}$, S.Torii $^{* 2}$, T.Makimura ${ }^{* 2}$, H.Niino ${ }^{* 3}$, K.Murakami $^{* 2}$, D.Nakamura ${ }^{* 1}$, A.Takahashi ${ }^{* 4}$, T.Okada $^{* 1}$ \\ ${ }^{* 1}$ Grad. Sch. of ISEE, Kyushu Univ., 744, Motooka, Nishi-ku, Fukuoka 819-0395, Japan \\ E-mail: okazaki@laserlab.ees.kyushu-u.ac.jp \\ ${ }^{* 2}$ Inst. of Applied Physics, Univ. of Tsukuba, 1-1-1, Tennoudai, Tsukuba, Ibaraki, 305-8577, Japan \\ ${ }^{* 3}$ National Institute of AIST, 1-1-1, Higashi, Tsukuba, Ibaraki, 305-8565, Japan \\ ${ }^{* 4}$ Grad. Sch. of Medical Sciences, Kyushu Univ. 3-1-1, Higashi-ku, Fukuoka, 812-8582, Japan
}

\begin{abstract}
A simple and productive micromachining method of a silica glass using a TEA (transversely excited atmospheric) $\mathrm{CO}_{2}$ laser $(10.6 \mu \mathrm{m})$ was investigated. A copper grid mask with a thickness of $2 \mu \mathrm{m}$, square apertures of $20 \times 20 \mu \mathrm{m}$ was attached to the silica glass surface, and it was irradiated by the TEA $\mathrm{CO}_{2}$ laser light. As a result, circular holes were formed on the silica glass surface at the center of each aperture in a spatial resolution down to the sub-wavelength scale. The minimum diameter of the hole was less than $3 \mu \mathrm{m}$, which is less than one third of the wavelength of the TEA CO${ }_{2}$ laser. A depth of the holes ranging from $100 \mathrm{~nm}$ to $600 \mathrm{~nm}$ could be micromachined on silica glass surface. The mechanism of the micromachining was discussed based on electric field distributions of the $\mathrm{CO}_{2}$ laser light by a 3D full-wave electromagnetic field simulation solver. The simulation results are in good agreement with the experimental results. It was found that the micromachining of the circular holes were caused by Fresnel diffraction of the $\mathrm{CO}_{2}$ laser light at the apertures.
\end{abstract}

DOI:10.2961/jlmn.2010.03.0016

key words: $\mathrm{CO}_{2}$ laser, metal mask, micromachining, silica glass, Fresnel diffraction,

\section{Introduction}

A micromachined silica glass is one of the most important materials in various fields of applications which include biochip sensor, micro optics and so on. Regarding the micromachining of silica glass, a laser processing is one of the most promising techniques because of a direct single step process. However, the laser micro-processing of transparent materials suffers from the difficulty of transferring photon energy of laser light to the materials. So far several techniques have been reported for laser processing of silica glass, including multi-photon processing using ultrafast laser ${ }^{[1]}$, laser-induced backside wet etching ${ }^{[2],[3]}$, ablation using vacuum ultra violet and extreme ultra violet light ${ }^{[4],[5]}$ sources, and so on. However, these techniques need relatively expensive laser systems. On the other hand, an infrared $\mathrm{CO}_{2}$ laser beam with a wavelength of $10.6 \mu \mathrm{m}$ has been widely used in industries because of its low cost and high power. In addition, it can be used for laser processing of the silica glass due to a high absorption coefficient for the wavelength of $10.6 \mu \mathrm{m}$. Meanwhile, a micro-processing with optical lenses is generally limited to several dozens of $\mu \mathrm{m}$ due to its longer wavelength resulting in limited diffraction.

In this work, we investigated the micro-processing at a sub-wavelength resolution with a help of Fresnel diffraction caused by a mask pattern irradiated by the $\mathrm{CO}_{2}$ laser beam.

\section{Experimental setup}

Fig. 1 shows the experimental configuration for a micromachining of a silica glass. A TEA $\mathrm{CO}_{2}$ laser which has a gain-switched peak pulse with a duration of $50 \mathrm{~ns}$ and a tail part of $1 \mu \mathrm{s}$ was used as an ablation laser. The silica glass was placed on an Al plate holder. A copper grid mask with a thickness of $2 \mu \mathrm{m}$, square apertures of $20 \times 20 \mu \mathrm{m}$ and bars of $5 \mu \mathrm{m}$ was attached to the silica glass surface as shown in Fig. 1. The silica glass was ablated by the $\mathrm{CO}_{2}$ laser at a fluence of $18 \mathrm{~J} / \mathrm{cm}^{2}$ and then the surface morphology was observed by a conventional optical microscope and an atomic force microscope (VN-8000M/8010M, KEYENCE).

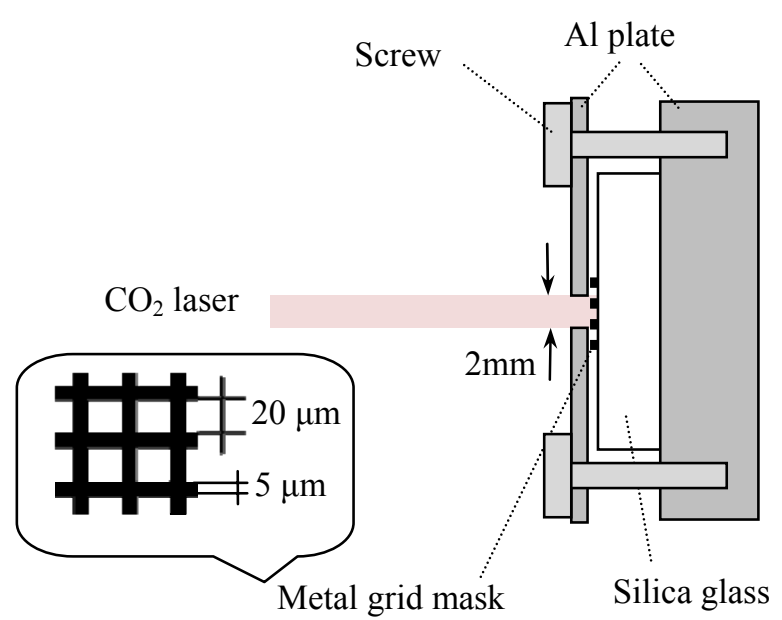

Fig.1 Experimental configuration 
3. Micromachining of silica glass with combination of TEA $\mathrm{CO}_{2}$ laser and metal grid mask

Fig. 2 (a) shows the optical microscope image of the irradiated surface after a single shot. Circular holes were formed on the silica glass surface at the center of each aperture by the $\mathrm{CO}_{2}$ laser light. Fig. 2 (b) shows a cross-sectional profile of the ablated surface along the red dashed line from Fig. 2 (a). This mesurment was performed by AFM. The diameter and the depth of the hole were about $7.3 \mu \mathrm{m}$ and $600 \mathrm{~nm}$, respectively. Furthermore, swellings with a height of about $50 \mathrm{~nm}$ were observed around the holes.

Fig. 3 (a) shows the optical microscope image of the silica glass surface after 10 shots. Circular holes without swellings were formed on the silica glass surface around the holes. Fig. 3 (b) shows the diameter and the depth of the hole were about $9.4 \mu \mathrm{m}$ and $500 \mathrm{~nm}$, respectively. The diameter of the holes after 10 shots is broader than that of single shot.

The depth of the holes is almost the same as that of single shot indicating that the ablation depth does not depend on the number of laser irradiation. This result seems to be attributed to the electric field distributions under the mask, as discussed in Sec. 4. Regarding the disappearance of the swelling, it is considered that the swellings around the holes gradually ablated by the 10 shots irradiation.

Fig. 4 (a) shows the optical microscope image observed at a different area on the same sample. There were small ablated points between the large holes where the place would be covered by the grid mask's bar. According to Fig. 4 (b), the diameter and the depth of the

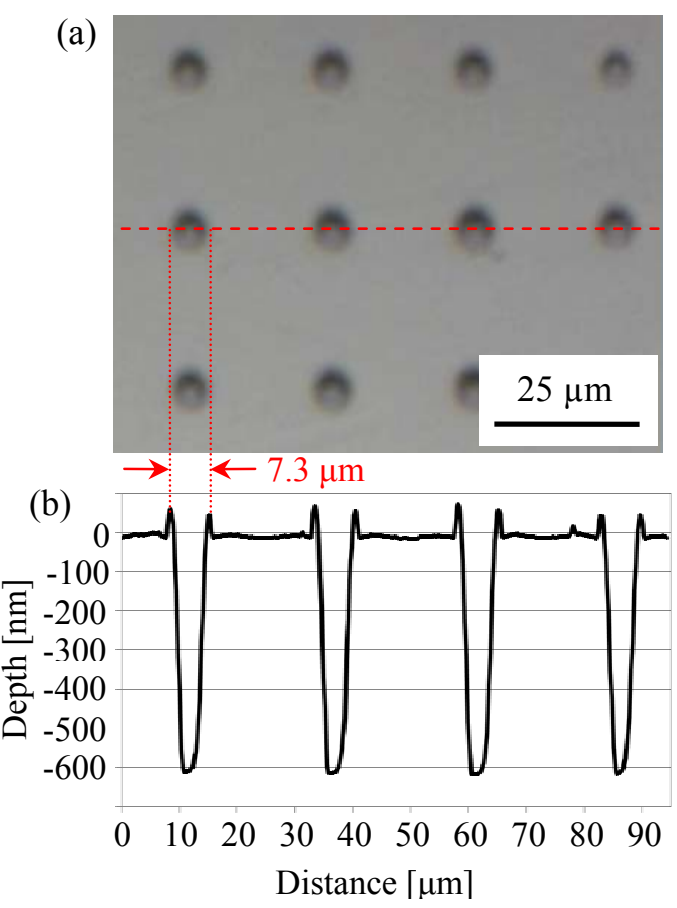

Fig. 2 (a) The optical microscope image of the ablated silica glass surface after a single shot. (b) The crosssectional profile of the ablated surface along the red-dashed line in Fig. 2 (a).
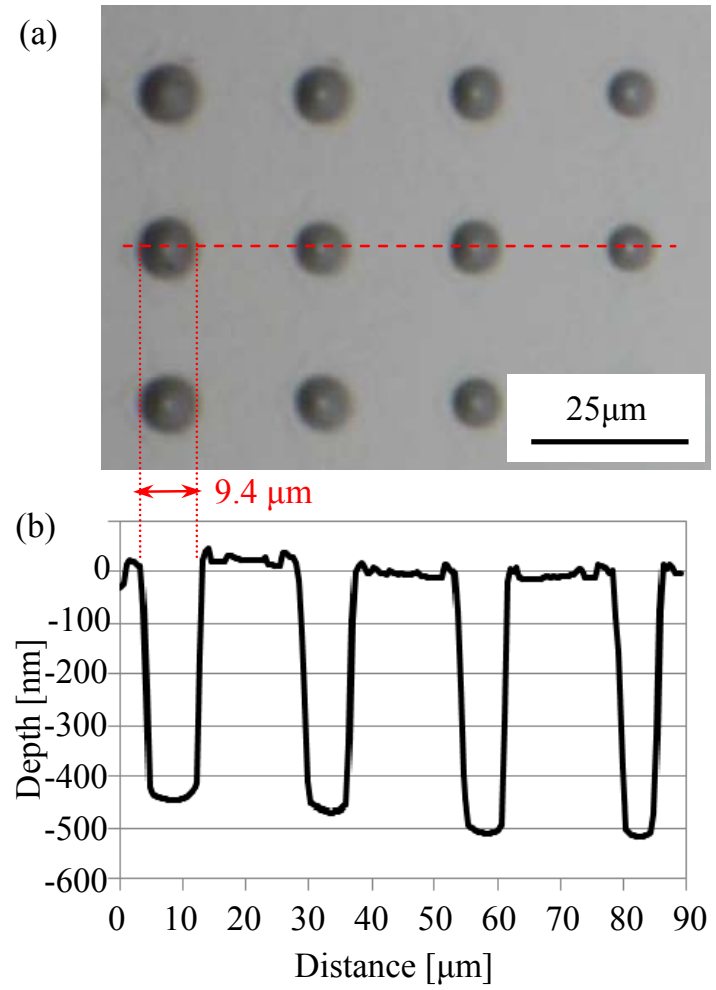

Fig. 3 (a) The optical microscope image of the ablated silica glass surface after 10 shots. (b) The crosssectional profile of the ablated surface along the red-dashed line in Fig. 3 (a).

(a)

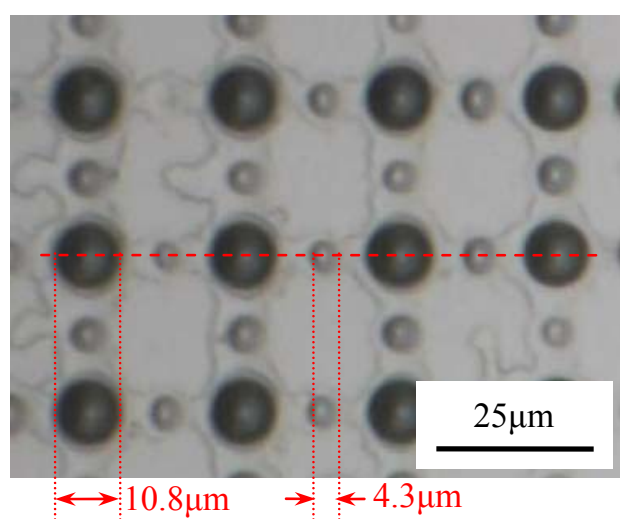

(b)

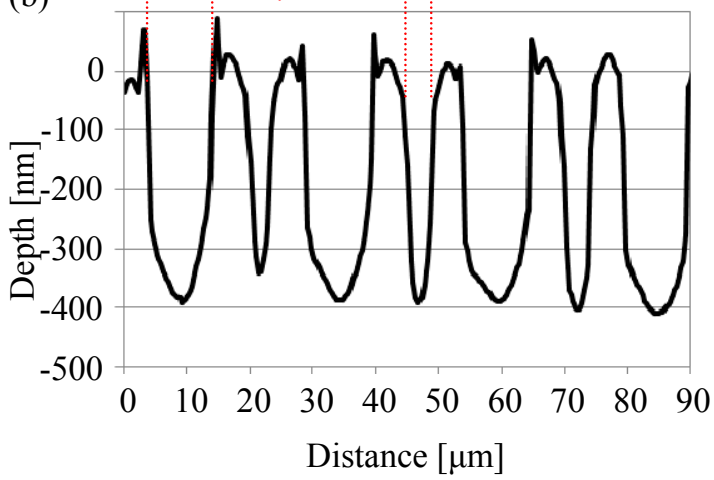

Fig. 4 (a) The optical microscope image of a different area on the same sample as Fig.3. (b) The cross-sectional profile of the ablated surface along the red-dashed line in Fig. 4(a). 
large holes were about $10.8 \mu \mathrm{m}$ and $400 \mathrm{~nm}$, respectively. In addition, a little ablated points were observed with the diameter of $4.3 \mu \mathrm{m}$ and the depth of $400 \mathrm{~nm}$.

Fig. 5 (a) shows the microscope image of the smallest ablated hole on the same silica glass as Fig. 3. The diameter and the depth of the smallest hole were $2.8 \mu \mathrm{m}$ and $25 \mathrm{~nm}$, respectively. It should be noted that the micromachining of the holes with a diameter less than one third of the laser light wavelength was succeeded in this technique.

(a)

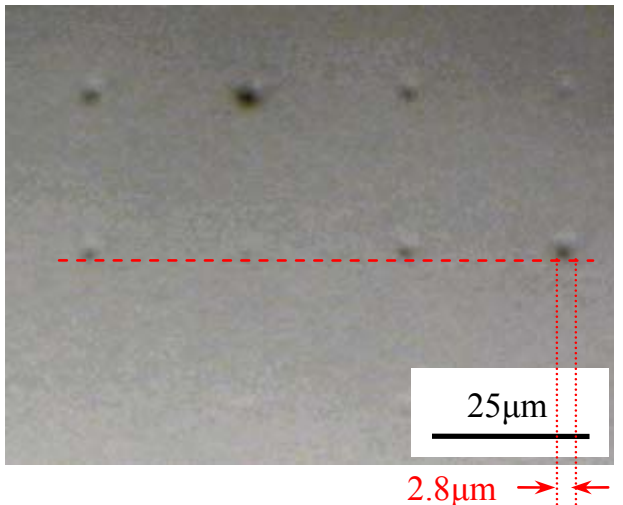

(b)

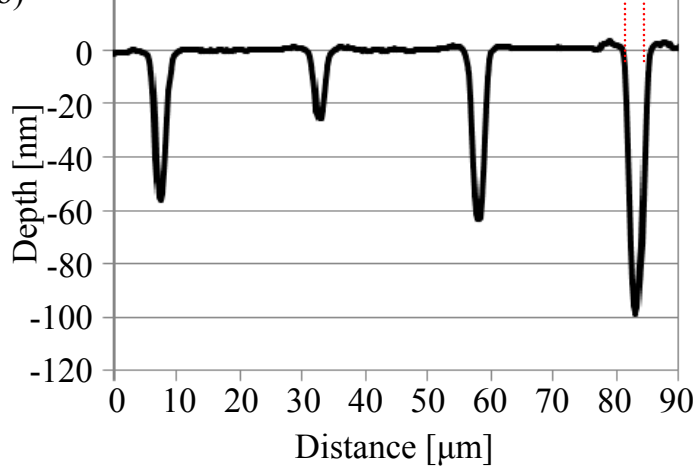

Fig. 5 (a) The optical microscope image of the smallest holes on the same sample. The cross-sectional profile of the ablated surface along the red-dashed line in Fig. 5 (a).

\section{Simulation of the electromagnetic field distributions under metal masks}

In this method, the circular holes were formed on the silica glass surface even though the mask had square apertures. It can be considered that the diffraction occurred because the wavelength of $\mathrm{CO}_{2}$ laser light was comparable with the mask apertures. For a theoretical consideration, the simulations of the electric field distributions were performed by the HFSS (High-Frequency Structure Simulator, ANSOFT ver. 11), a commercial full-wave electromagnetic solver.

Fig. 6 shows the calculated electric field distributions around the copper grid mask with a thickness of $2 \mu \mathrm{m}$, square apertures of $20 \times 20 \mu \mathrm{m}$ and bars of $5 \mu \mathrm{m}$. An incident light is a plane wave with the wavelength of 10.6 $\mu \mathrm{m}$ propagating along the $\mathrm{z}$-axis and linearly polarized in $\mathrm{x}$-axis. The result from $\mathrm{x}-\mathrm{z}$ plane shows that hot spots appeared about $9 \mu \mathrm{m}$ below the mask. We can see the circular hot spots at the center of each aperture from the $\mathrm{x}-\mathrm{y}$ plane. We believe that the circular holes on the silica glass beyond the diffraction limit were formed by the hot spots. Furthermore, weak hot spots also appeared under the mask's bars in the $y-z$ plane. Since these interferences cannot be seen in the $x-z$ plane, it would depend on the polarization state. This simulation results can be compared with the experimental results as shown in Fig. 4 (b) where small ablated holes appeared at the middle of the large holes. In the experiment, a little ablated holes were formed not only along $\mathrm{y}$-axis, but also along $\mathrm{x}$-axis due to the random polarization of $\mathrm{CO}_{2}$ laser light.

The simulations were also performed to investigate the relation between the effect of the hot spots caused by the single and the multiple apertures. In this simulation, the mask was a copper with a thickness of $2 \mu \mathrm{m}$ and a single aperture of $20 \times 20 \mu \mathrm{m}$. The incident light is the same as in Fig. 6. The simulation results are shown in Fig. 7. A single hot spot appeared $9 \mu \mathrm{m}$ below the mask. It was found that the hot spot was formed not only by multiple apertures of the mask, but also by a single aperture. On the other hand, no weak hot spots were observed in Fig. 7 as compared to Fig. 6. This indicates that the weak hot spots were responsible for the interferences of lights through the
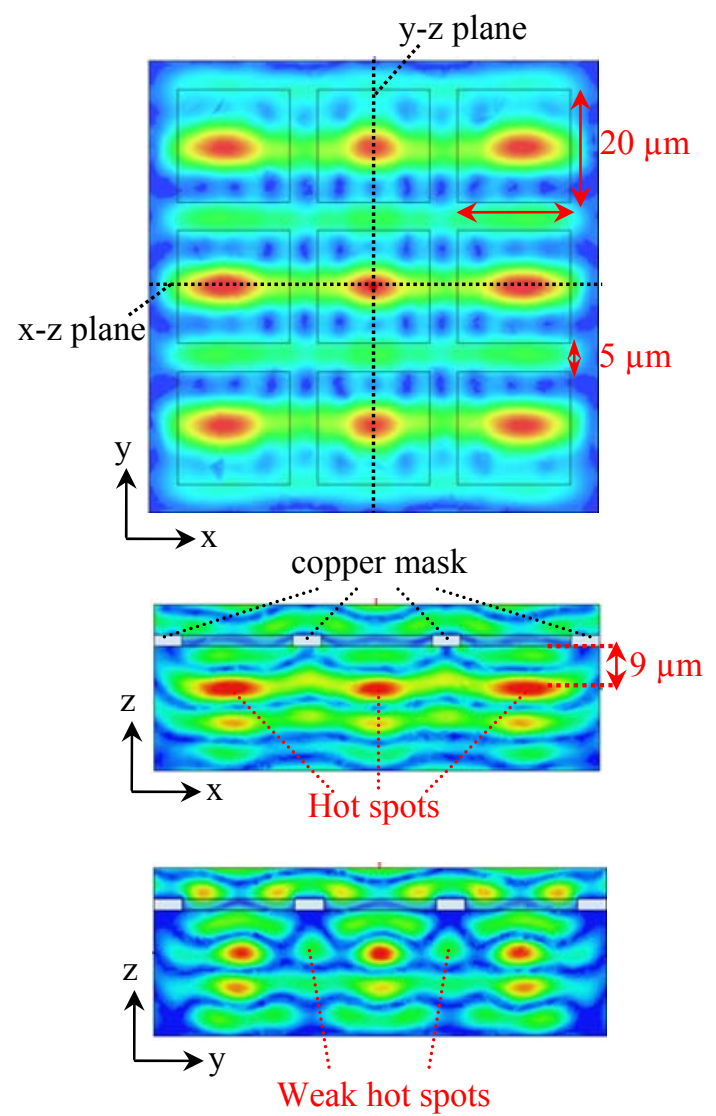

Fig. 6 Calculated electric field distributions around the copper grid mask with multiple square apertures. Incident light is a plane wave with the wavelength of $10.6 \mu \mathrm{m}$ propagating along -z-axis and linearly polarized in the x-axis. The hot spots appeared at the center of each aperture about $9 \mathrm{um}$ below the mask. 
JLMN-Journal of Laser Micro/Nanoengineering Vol. 5, No. 3, 2010

neighboring apertures. Though the hot spots were appeared $9 \mu \mathrm{m}$ below the mask, the depth of the hole in the experiments was about $600 \mathrm{~nm}$ as shown in Fig. 2. The difference between the simulation and the experiment would be caused by the gap distance between the mask and the silica glass in the experiment. It is difficult to control the gap distance in the present experiment and there may be some distance inevitably. In Fig. 3, 4 and 5, variation in the size of the holes were observed across the surface. It is thought that this is mainly due to the non-uniform gap distance. Thus the gap distance plays a major role in the quality of the micromachining, hence the well-controlled gap distance could realize a deeper micromachining of the silica glass up to a depth of $9 \mu \mathrm{m}$.

These simulation results would be in good agreement with the experimental results, except for the polarization of the $\mathrm{CO}_{2}$ laser light. In this method, the electric field distributions of the light through the mask are defined by the mask aperture size, shape, thickness and so on. This indicates that various-shaped micromachining could be formed on the silica glass by designing the mask shape based on the simulation.

For instance, the electric field distributions in changing the mask aperture into rectangles are shown in Fig. 8. The mask has a thickness of $2 \mu \mathrm{m}$, aperture size of $20 \times 70 \mu \mathrm{m}$ whose long axis is directed toward the $\mathrm{x}$-axis, and the incident light is also the same as Fig. 6. As the mask aperture is rectangle, the hot spots appeared in a form of a line along the direction of the long axis at about $15 \mu \mathrm{m}$ below the mask. In this way, the simple and productive silica glass micromachining would be realized by designing the mask which gave optimum and specific electric field distributions.

\section{Conclusions}

A simple and productive micromachining method for a silica glass has been investigated. It is capable of

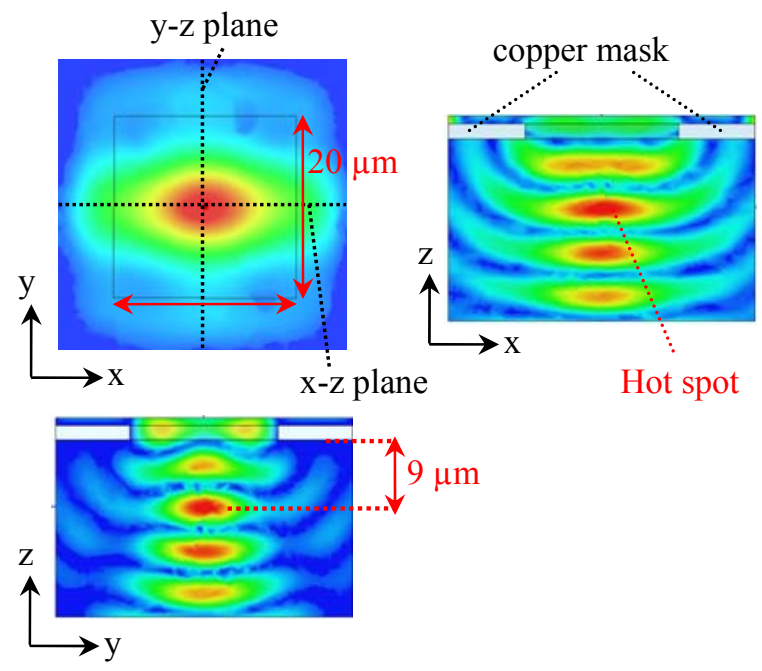

Fig. 7 Calculated electric field distributions around the copper grid mask with a single square aperture. Incident light is the same as Fig. 6. The hot spot was formed even in a single aperture. micromachining of the silica glass at sub-wavelength by

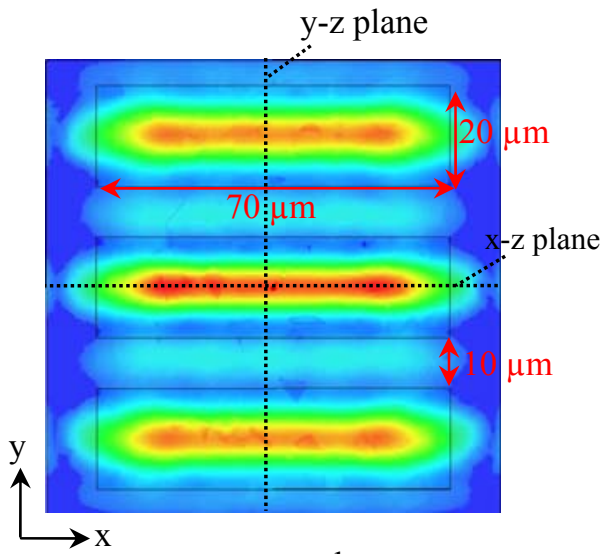

copper mask $\because \ddots$
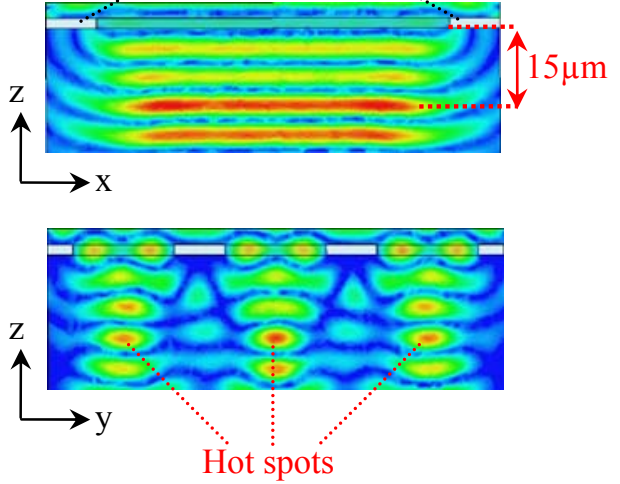

Fig. 8 Calculated electric field distributions around the copper grid mask with multiple and rectangular apertures. Incident laser is the same as Fig. 6. Linearly-shaped hot spots appeared at the center of each aperture about $15 \mu \mathrm{m}$ below the mask.

modified electric field distributions of $\mathrm{CO}_{2}$ laser light under a copper mask in Fresnel diffraction region. A micro hole of diameter ranging from $2.8 \mu \mathrm{m}$ to $10.8 \mu \mathrm{m}$ and a depth of $100 \mathrm{~nm}$ to $600 \mathrm{~nm}$ could be micromachined on silica glass surface. The simulation of the electric field distributions were performed for both single and multiple apertures and they are in good agreement with the experimental results. The simple and productive micromachining of the silica glass would be realized by designing the mask shape based on the simulation.

\section{References}

[1] Fei He, Ya Cheng, Zhizhan Xu, Yang Liao, Jian Xu, Haiyi Sun, Chen Wang, Zenghui Zhou, Koji Sugioka, Katsumi Midorikawa, Yonghao Xu, and Xianfeng Chen: Optics Lett. 35 (2010) 282

[2] Hiroyuki Niino, Yoshizo Kawaguchi, Tadatake Sato, Aiko Narazaki, Ryozo Kurosaki: Appl. Sur. Sci. 253 (2007) 8287

[3] Tadatake Sato, Ryozo Kurosaki, Aiko Narazaki, Yoshizo Kawaguchi, Hiroyuki Niino: Appl. Phys. A 101 (2010) 319

[4] Tetsuya Makimura, Shinjo Mitani, Youichi Kenmotsu, and Kouichi Murakami: Appl.Phys.Lett. 85 (2004) 1274

[5] Tetsuya Makimura, Hisao Miyamoto, Youichi Kenmotsu, and Kouichi Murakami: Appl.Phys.Lett. 86 (2005) 103111 